\title{
Perceptions, usages et vulnérabilité des ressources végétales ligneuses dans le Sud-Ouest du Burkina Faso
}

\author{
Lassina TRAORE * Issaka OUEDRAOGO, Amadé OUEDRAOGO et \\ Adjima THIOMBIANO
}

Laboratoire de Biologie et Ecologie Végétales, UFR/SVT, Université de Ouagadougou, 09 BP 848

Ouagadougou 09, Burkina Faso.

*Auteur correspondant ; E-mail : ltraorej@gmail.com; Tel : +22676 630533

\section{RESUME}

Au Burkina Faso, les populations rurales dépendent fortement des ressources végétales ligneuses pour la satisfaction de leurs besoins quotidiens. Dans un contexte de pression anthropique croissante, l'évaluation de la vulnérabilité des espèces s'avère un impératif majeur pour permettre la préservation et la gestion durable de ces ressources. Pour ce faire, des enquêtes semi-structurées ont été menées chez les Sénoufo, un des groupes ethniques dominants du Sud-Ouest du Burkina Faso. La vulnérabilité des espèces citées par les personnes enquêtées a été évaluée sur la base d'un indice de vulnérabilité (IV) établi à partir de six paramètres. Au total, 73 espèces ont été citées pour lesquelles l'artisanat, le bois de feu et la construction se révèlent être les principales causes de vulnérabilité. Certains aspects des croyances traditionnelles s'avèrent cependant favorables à la conservation des ressources végétales ; ainsi, par exemple, certaines espèces sont interdites à la combustion. Cette étude montre que la combinaison des inventaires de terrain et des enquêtes ethnobotaniques est indispensable comme approche fiable pour l'identification des espèces menacées.

(C) 2011 International Formulae Group. All rights reserved.

Mots clés: conservation, espèces ligneuses, indice de vulnérabilité, tradition, utilisations.

\section{INTRODUCTION}

La dégradation continue du couvert végétal ouest africain est en partie due à la croissance démographique et aux facteurs climatiques (Wezel and Haigis, 2000). Cette dégradation des écosystèmes et de la richesse en espèces est une des conséquences de l'aridité du climat qui est devenue un phénomène réel en zone soudanienne (Thiombiano, 2005). La fragilité du milieu soudanien est d'autant plus perceptible lorsque les facteurs anthropiques comme les feux de brousse et le surpâturage s'ajoutent aux facteurs climatiques. La diminution des ressources ligneuses notamment, affecte tous les domaines de la vie des populations locales : essentiellement l'alimentation, l'énergie, la construction, la médecine et l'artisanat (Wezel and Lykke, 2006). Pour des raisons diverses, l'ampleur de cette dégradation de la végétation varie d'une région à une autre. De récentes études menées sur la connaissance et la perception des populations locales dans le Sahel ont permis, non seulement de connaître de nombreuses utilisations des espèces ligneuses, mais aussi 
de comprendre l'ampleur des changements dans la végétation pendant ces dernières décennies (Hahn-Hadjali et Thiombiano, 2000; Kristensen and Balslev, 2003; Lykke et al., 2004; Wezel and Lykke, 2006). Tous ces travaux ont révélé un certain nombre d'espèces dont la disparition est perçue par les populations locales.

$\mathrm{Si}$ les phénomènes climatiques et anthropiques de la dégradation des ressources végétales ont été abondamment décrits dans la littérature, il existe cependant très peu d'études détaillées sur la vulnérabilité des espèces en fonction des zones géographiques, notamment dans la région soudanienne. En outre, les travaux antérieurs sur l'ethnobotanique (Kristensen and Balslev, 2003; Lykke et al., 2004; Belem et al., 2007; 2008) n'ont pas clairement évalué l'impact écologique des usages sur la base d'indicateurs de vulnérabilité des espèces utilisées. Or, de manière globale, l'impact écologique de l'exploitation des ressources forestières est non seulement fonction de la composition floristique des formations végétales, de la nature et de l'intensité de la récolte des produits (Peters, 1997), mais aussi des facteurs sociaux et économiques (Betti, 2001). La vulnérabilité correspond donc ici au degré d'exposition aux risques de réduction ou de disparition de certaines espèces végétales occasionnés par les modes de prélèvements inappropriés dans un environnement soumis à une pression anthropique croissante et à des variations climatiques. Dans la perspective d'un développement social axé sur la lutte contre la pauvreté, la connaissance des espèces vulnérables représente une étape importante pour intégrer les besoins des populations dans les prises de décision concernant la gestion durable des ressources végétales. La performance des stratégies à mettre en place pour réduire ou freiner la perte des espèces végétales est étroitement liée à la fiabilité des outils de leur mise en œuvre. Dès lors, l'évaluation de la vulnérabilité en prenant en compte les facteurs anthropiques, s'avère un impératif majeur de la réussite des actions de gestion durable des ressources végétales des pays en voie de développement. L'une des rares investigations sur la vulnérabilité des plantes, semble être les récents travaux de Ouédraogo-Belem (2008). Ces travaux ont permis d'évaluer et de dresser une liste de plantes vulnérables de la réserve de la Biosphère de la Mare aux Hippopotames du Burkina Faso, pour une meilleure gestion de ce patrimoine naturel. Pour notre part, nous avons axé notre étude sur les ligneux, qui constituent les composantes permanentes des savanes avec pour objectifs :

- d'appréhender la perception locale de la disponibilité des ressources végétales ;

- de déterminer les espèces préférées par les populations ;

- d'évaluer la vulnérabilité des espèces dans un contexte d'utilisation quotidienne.

Elle contribuera, d'une part, à documenter les nombreuses utilisations des ligneux au Sahel et, d'autre part à mettre à la disposition des acteurs chargés de la mise en œuvre des politiques de gestion des ressources naturelles, des données fiables pour une meilleure préservation des espèces végétales dont l'état de conservation est précaire.

\section{MATERIEL ET METHODES}

\section{Zone d'étude}

L'étude a été conduite dans trois villages (Dakoro, Niankorodougou et Kankalaba) de la province de la Léraba (Figure 1) située dans le secteur phytogéographique sud-soudanien du Burkina Faso (Fontes et Guinko, 1995). La province fait partie de la région des Cascades caractérisée par deux grandes saisons (une saison humide qui s'étale d'avril à octobre et une saison sèche qui s'étale de novembre à mars). Les températures moyennes annuelles varient entre $17{ }^{\circ} \mathrm{C}$ (minimum) et $36{ }^{\circ} \mathrm{C}$ (maximum). Cette région est l'une des plus arrosée du pays avec une moyenne pluviométrique de 1071,09 $\mathrm{mm}$ sur une période de dix ans (1999-2008) (Figure 2). La végétation est principalement composée de 
savanes et de forêts claires (Taïta, 2003). Les Cascades abritent seulement $3,8 \%$ de la population totale du Burkina Faso, avec 14017262 habitants (INSD, 2008), ce qui fait d'elle la région la moins peuplée du pays. Les principales activités sont l'agriculture, l'élevage et l'artisanat. Au Burkina Faso, les Sénoufo se rencontrent dans deux provinces, le Kénédougou et la Léraba. Ce peuple se rencontre près des lignes frontalières entre le Burkina, la Côte d'Ivoire et le Mali. La population Sénoufo est essentiellement rurale. Traditionnellement, le Sénoufo est à la fois fétichiste, animiste et naturaliste (Ouattara, 2008). Fétichiste, il est profondément attaché au culte des fétiches et des idoles qui sont une représentation des dieux protecteurs. Animiste, il croit à l'esprit des morts et à l'existence d'une vie après la mort. Il est naturaliste parce qu'il obéit aux forces de la nature auxquelles il voue adoration. Les Sénoufo pensent que chaque élément de la nature, à savoir le ciel, la terre, le feu, le vent, les animaux, les arbres, etc. est doté d'un souffle. Les Sénoufo entretiennent une forte relation avec les esprits des ancêtres. Pour ce faire, ils communiquent régulièrement avec le monde des divinités et des ancêtres à qui ils doivent obéissance et dévotion.

\section{Récolte des données}

Les informations ont été obtenues grâce à des interviews semi-structurées. L'âge des informateurs varie entre 30 et 75 ans. Les informateurs ont été enquêtés individuellement avec l'aide de guides locaux. Au total, 60 personnes ont été interviewées (20 informateurs par village). Le questionnaire concerne l'usage des ressources végétales ligneuses et se rapporte à toutes les parties de la plante qui sont utilisées, les domaines d'utilisation, le mode de collecte, le stade de développement de l'organe ou produit végétal utilisé, le point de vue de l'interviewé sur l'état d'abondance des espèces utilisées, les éventuelles causes de la rareté des ressources végétales et des propositions de stratégies de conservation des espèces utiles.

Dans l'objectif de confronter les réponses des informateurs aux données de terrain, des relevés d'inventaires dendrométriques ont été effectués de manière aléatoire. La collecte des données a visé à calculer l'importance écologique des espèces, à travers les indices de valeur d'importance et de rareté. L'inventaire a concerné les arbres et arbustes dont le diamètre à $1,30 \mathrm{~m}$ du sol est supérieur ou égalé à $5 \mathrm{~cm}$ (Ouédraogo, 2006) correspondant aux individus exploitables. Les relevés ont été effectués dans 50 parcelles de $50 \mathrm{~m} \times 20 \mathrm{~m}$.

\section{Analyse des données}

L'importance écologique des espèces a été appréciée à partir de l'Indice de Valeur d'Importance (IVI) qui permet de mieux apprécier leur importance dans une communauté végétale. Cet indice, pour une espèce, se définit comme la somme de sa dominance relative, de sa densité relative et de sa fréquence relative qui se calculent comme suit :

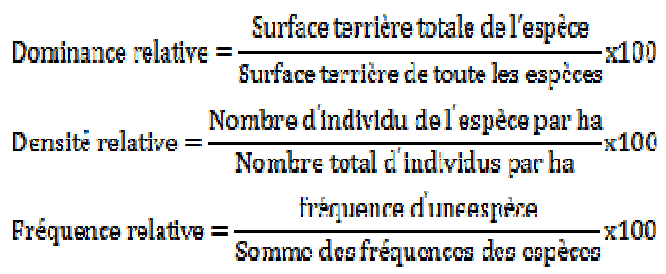

La disponibilité des espèces utilisées a été appréciée à partir des relevés réalisés sur 50 parcelles. Pour ce faire, un indice de rareté des espèces (Rarity-weighted Richness Index) a été calculé suivant l'équation de Géhu and Géhu (1980) in Kokou et al. (2005): RI = [1$(\mathrm{ni} / \mathrm{N})]$ x 100 , avec $R I=$ l'indice de rareté, $n i=$ nombre de relevés dans lesquels l'espèce $i$ est présente et $N=$ nombre total de relevés. Dans des travaux récents, Kokou et al. (2005) et Adomou et al. (2005) ont utilisé cet indice en retenant le seuil de $80 \%$ pour séparer les espèces rares des espèces fréquentes dans la zone phytogéographique subéquatoriale. Dans notre cas, en tenant compte du contexte soudanien, des seuils d'interprétation de 
l'indice de rareté ont été retenus pour prendre en compte les espèces de fréquences moyennes. C'est ainsi que lorsque RI $<60 \%$, les espèces sont dites très fréquentes dans les formations végétales. Celles dont $60 \leq \mathrm{RI}<$ $80 \%$ sont moyennement fréquentes. Celles dont RI $\geq 80 \%$ sont dites rares.

L'échelle de vulnérabilité qui comportant trois niveaux, de 1 à 3 , proposée par Betti (2001), a été utilisée pour calculer la vulnérabilité des espèces. Une valeur de 1 désigne une espèce peu vulnérable pour les paramètres indiqués, une valeur de 2 représente une vulnérabilité moyenne et une valeur de 3 caractérise une espèce très vulnérable. Les indices de vulnérabilité ont été calculés à partir des paramètres suivants :

Les fréquences d'utilisations (N1). La vulnérabilité d'une espèce ligneuse augmente avec sa fréquence d'utilisation (popularité).

Les types d'usages des espèces (N2). La vulnérabilité d'une espèce ligneuse augmente avec le nombre d'usage dans lequel la plante est sollicitée. Plus le nombre d'usage augmente, plus la pression s'accroît sur l'individu qui est sollicité.

Les organes utilisés (N3). La vulnérabilité d'une plante augmente selon que l'organe végétal prélevé se régénère facilement ou non. La récolte d'écorce, de tissus de la tige et de racines tue presque toujours les arbres (Betti, 2001), en revanche celle de latex, de fruits et de feuilles ne conduit pas nécessairement à la mort des arbres adultes et ne modifie pas radicalement la distribution des classes d'âge de la population (Peters, 1997). La récolte sporadique de quelques fruits aura sans doute moins d'effet sur la stabilité à long terme des populations de ligneux ainsi exploités. En revanche, une récolte intensive des fruits ou des graines par exemple, peut entraîner une diminution progressive des ligneux qui les produit. Ce phénomène est observé pour Prosopis africana où la récolte excessive des gousses a sérieusement réduit la régénération naturelle dans les régions sahéliennes de l'Afrique de l'Ouest (Weber et al., 2008).
Le mode de prélèvement (N4). Les principaux modes de prélèvements retenus sont le ramassage, la cueillette et la coupe. Les populations des individus qui sont partiellement élaguées seront moins vulnérables que celles qui sont entièrement abattues. De même, les individus dont on cueille les organes végétaux seront traumatisés et donc plus vulnérables que ceux dont on ramasse aisément les parties tombées sur le sol.

Le stade de développement de l'organe prélevé (N5). Les stades de développement utilisés dans le cadre de ce travail, sont ceux retenus par Betti (2001) : jeune, adulte, vieux ou sénescent. Les individus dont les organes sont cueillis tôt, avant maturité, seront plus vulnérables du fait du traumatisme subi.

La fréquence relative des espèces dans les relevés (N6). La vulnérabilité d'une espèce diminue avec sa fréquence dans le milieu (nombre de relevés où l'espèce est présente). Une espèce, à fréquence relativement élevée dans les formations végétales, sera moins vulnérable que celle à faible fréquence.

Lorsque plusieurs parties d'une plante sont sollicitées dans un usage, seule la partie qui a la plus grande valeur issue de l'échelle de vulnérabilité est prise en compte dans le calcul des indices. Toutes les espèces utilisées ont pu être observées directement sur le terrain. Dès lors que l'existence de toutes les espèces issues des enquêtes est effective, celles qui n'ont pas été rencontrées dans les relevés dendrométriques que nous avons réalisés, ont été considérées comme espèces à faible fréquence dans le milieu.

La fréquence d'utilisation relative (N1) d'une espèce $i$ dans un usage $j$ est calculée selon la formule suivante : N1 = npij/ntpe $\mathrm{x}$ 100 avec npij= nombre de personnes ayant cité l'espèce $i$ dans un usage $j$; ntpe $=$ nombre total des informateurs. Les valeurs de N3, N4 et N5 varient de 1 à 3 suivant le type d'organe, le mode de prélèvement et le stade de développement de l'organe végétal (Tableau 1). La fréquence relative (Fr) (N6) a été calculée selon la formule suivante: 
L. TRAORE et al. /Int. J. Biol. Chem. Sci. 5(1): 258-278, 2011

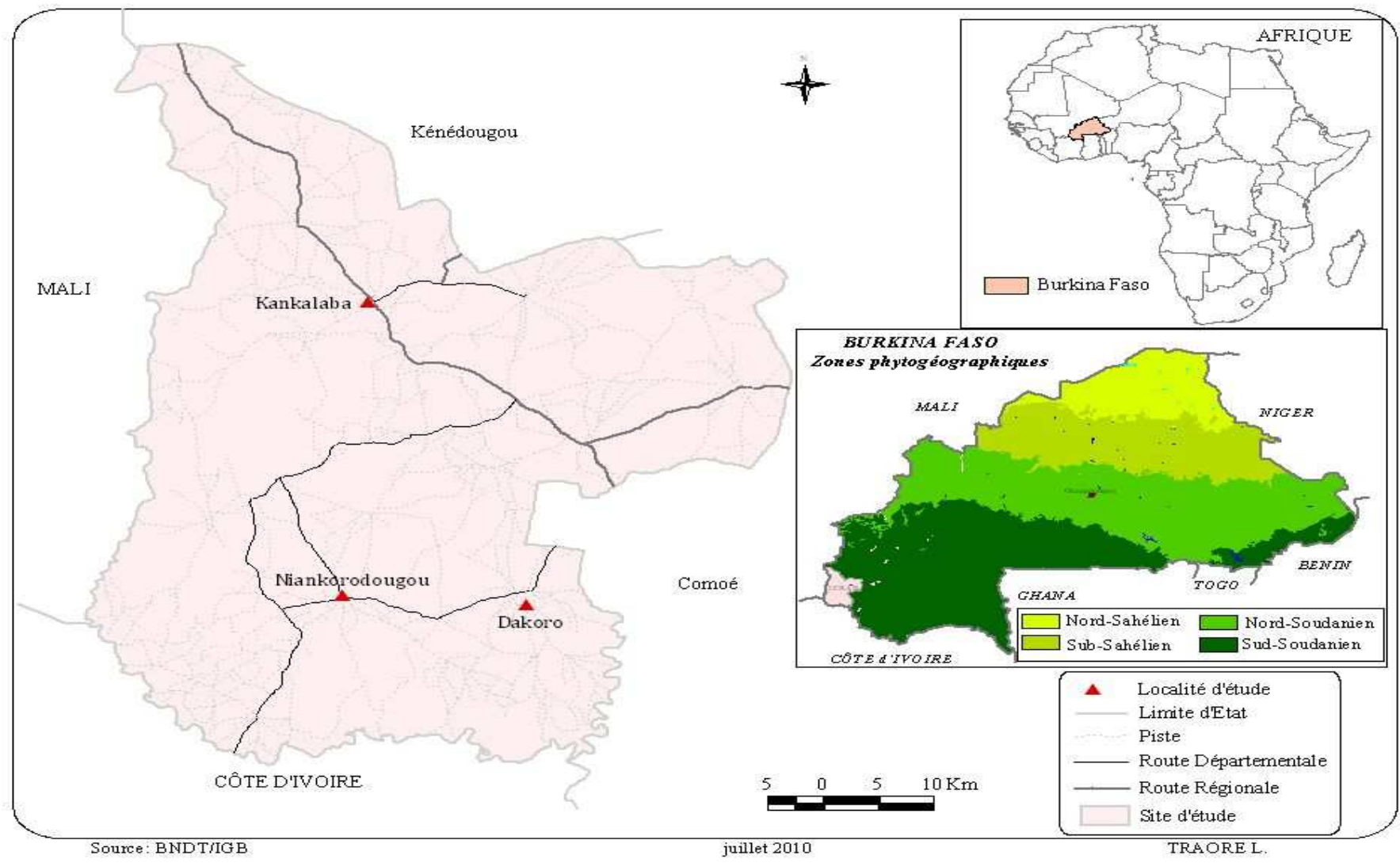

Figure 1: Carte de localisation de la zone d'étude. 


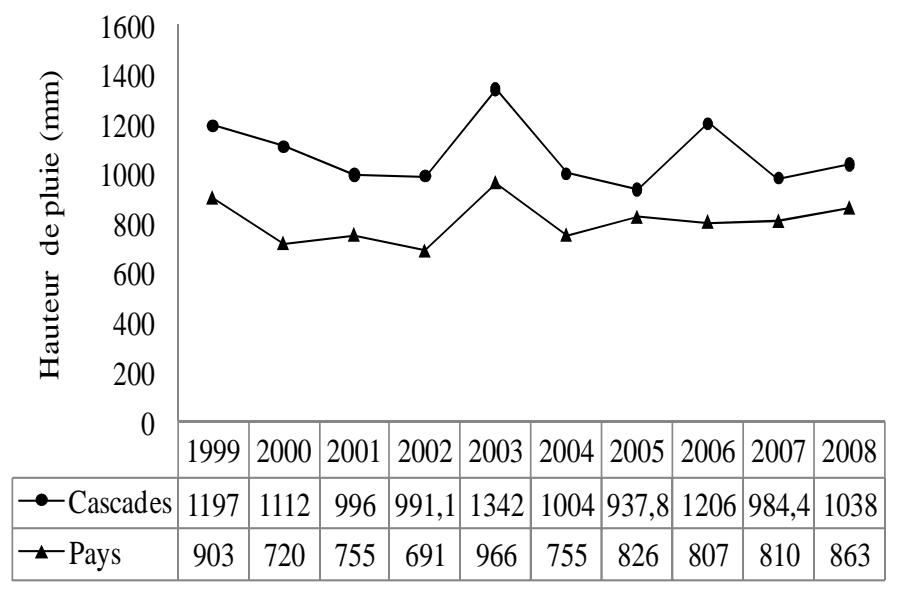

Figure 2: Pluviométrie moyenne comparée de la région des Cascades à celle du Burkina Faso. Source : Direction de l'Aviation et de la Météorologie 2010.

Tableau 1: Paramètres importants pris en compte pour le calcul d'indice de vulnérabilité (inspirée de Betti, 2001).

\begin{tabular}{|c|c|c|c|}
\hline \multirow[t]{2}{*}{ Paramètres } & \multicolumn{3}{|c|}{ Vulnérabilité à une exploitation incontrôlée } \\
\hline & Faible (échelle = 1) & Moyenne (échelle $=2$ ) & Forte (échelle $=3$ ) \\
\hline $\begin{array}{l}\text { 1. Fréquence } \\
\text { d'utilisation }\end{array}$ & Faible F.U< $20 \%$ & $\begin{array}{l}\text { Moyenne } 20 \% \leq \text { F.U }< \\
60 \%\end{array}$ & Elevée F.U $\geq 60 \%$ \\
\hline 2. Nombre d'usages & $\mathrm{Nu}<2$ & $2 \leq \mathrm{Nu} \leq 4$ & $\mathrm{Nu} \geq 5$ \\
\hline $\begin{array}{l}\text { 3. Organe végétal } \\
\text { utilisé }\end{array}$ & Feuille, latex & Fruit, branche & $\begin{array}{l}\text { Bois, graine, écorce, } \\
\text { racine, fleur }\end{array}$ \\
\hline 4. Mode de collecte & Ramassage & - & Cueillette, coupe \\
\hline $\begin{array}{l}\text { 5. Stade de } \\
\text { développement }\end{array}$ & Vieux ou sénescent & Adulte & Jeune \\
\hline $\begin{array}{l}6 . \text { Fréquence } \\
\text { relative }\end{array}$ & $\mathrm{Fr} \geq 2 / 3 \mathrm{Fm}$ & $1 / 3 \mathrm{Fm} \leq \mathrm{Fr}<2 / 3 \mathrm{Fm}$ & Fr $<1 / 3 \mathrm{Fm}$ \\
\hline
\end{tabular}

$\mathrm{Fr}=\mathrm{ni} / \mathrm{N}$ x 100 avec $\mathrm{Fr}:$ fréquence $(\%) \mathrm{de}$ l'espèce $\mathrm{i} ;$ ni : nombre de relevés où l'espèce $\mathrm{i}$ est présente; $\mathrm{N}$ : nombre total de relevés. L'échelle utilisée est relative à la plus grande fréquence relative obtenue, désignée par Fm (Tableau 1). Ainsi, le calcul de l'indice de vulnérabilité de l'espèce $i\left(I V_{i}\right)$ suit la formule suivante : $I V i=\mathrm{N} / 6$ avec $\mathrm{N}=\mathrm{N} 1+\mathrm{N} 2+\mathrm{N} 3+$ $\mathrm{N} 4+\mathrm{N} 5+\mathrm{N} 6 . \mathrm{Si} I V i<2$, la plante est dite faiblement vulnérable; si $2 \leq I V i<2,5$, la plante est dite moyennement vulnérable; si $I V i \geq 2,5$, la plante est dite très vulnérable. La nomenclature utilisée pour la taxonomie est IPNI (The International Plant Names Index).

\section{RESULTATS}

Utilisations des plantes ligneuses

Un total de 73 espèces ligneuses réparties en 64 genres et 31 familles a été recensé lors des enquêtes. 94 espèces 
ligneuses reparties en 76 genres et 34 familles sont issues des inventaires (Figure 3). Le nombre d'espèces utilisées par les Sénoufo représente $77,65 \%$ du nombre d'espèces inventoriées dans les relevés. On note une prédominance des Fabaceae-Mimosoideae, suivi des Combretaceae, des Caesalpiniaceae, des Rubiaceae au niveau des espèces inventoriées, tandis qu'au niveau des utilisations, ce sont les Combretaceae qui dominent, suivi des Fabaceae-Mimosoideae, des Caesalpiniaceae et des Rubiaceae (Figure 4). Les familles qui ne comptent qu'une seule espèce sont regroupées sous le nom de « autres familles ». Les usages sont guidés par la disponibilité des ressources car les taxons les plus sollicités sont aussi ceux qui sont les mieux représentés dans les formations végétales. Les produits ou organes utilisés sont le bois $(30,58 \%)$, les feuilles $(23,14 \%)$, les fruits $(19,01 \%)$, les branches $(15,70 \%)$, les écorces $(6,70 \%)$, les graines $(4,96 \%)$, les racines $(4,13 \%)$, la pulpe $(1,65 \%)$ et le calice $(0,83 \%)$.

Les principaux modes de récolte des organes végétaux sont la cueillette et la coupe. Les fruits, les feuilles et les fleurs sont cueillis généralement à la main. Le bois, les branches et les écorces sont prélevés à l'aide d'une machette ou d'une hache. Les populations se soucient très peu des dommages causés par le prélèvement. Les 73 espèces sont sollicitées dans six catégories d'utilisations : l'alimentation, l'artisanat, la construction, le bois de feu, le fourrage et la médecine traditionnelle (Tableau 2). Les espèces les plus appréciées en alimentation sont Vitellaria paradoxa, Parkia biglobosa, Saba senegalensis et Annona senegalensis. Les fruits et graines de ces espèces sont consommés directement ou préparés et sont très bien appréciés des Sénoufo.

Dans le domaine du bois énergie, les Sénoufo reconnaissent que toutes les espèces peuvent être utilisées en combustion, à l'exception de celles qui sont interdites pour des raisons de croyances traditionnelles. Les espèces appréciées pour le bois de feu sont Detarium microcarpum, Anogeissus leiocarpa, Prosopis africana, Pterocarpus erinaceus, Combretum collinum, Hymnocardia acida et Pericopsis laxiflora. 14 espèces sont interdites dans la combustion dont les principales en termes de taux de citations sont Combretum molle (97,78\%) Diospyros mespiliformis (90\%) et Afzelia africana $(53,33 \%)$. Les autres espèces ont des fréquences de citation assez faibles (Tableau 3). Les raisons d'interdiction, entre autres, évoquées sont le totem, la destruction de la famille, l'interdiction par les ancêtres, la perte de la fécondité, le lieu de culte.

Dans le domaine de la construction, les espèces sont choisies sur la base de la résistance de leur bois aux termites et aux autres insectes foreurs. Les espèces les plus utilisées dans la construction des habitats sont Anogeissus leiocarpa, Prosopis africana, Pterocarpus erinaceus. Les espèces épineuses (Acacia dudgeonii, Zanthoxylum zanthoxylö̈des) et d'autres espèces comme Gardenia aqualla, Gardenia erubescens, Gardenia ternifolia) sont utilisées pour la construction de parcs à bétail qui sont des abris temporaires.

Dans le domaine du fourrage, Afzelia africana et Pterocarpus erinaceus sont les plus utilisées, car selon $86,5 \%$ des interviewés, ces espèces sont bien appétées par les animaux. Les individus de ces espèces sont annuellement émondés en saison sèche pour nourrir le bétail.

Dans le domaine de l'artisanat utilitaire, plusieurs espèces sont utilisées (Tableau 2). Le choix de l'espèce tient compte de la valeur accordée à l'objet confectionné. Selon qu'il s'agisse du mortier, du pilon ou du tabouret, le choix de l'espèce varie. Ainsi, Prosopis africana, Pterocarpus erinaceus sont utilisées pour confectionner le mortier et le pilon grâce à la résistance de leur bois, tandis que Ficus sycomorus est utilisée dans la confection du tabouret. 
Dans le domaine de la médecine traditionnelle, les remèdes les plus cités sont ceux contre le paludisme. Anogeissus leiocarpa avec $72,5 \%$ de citations, est très sollicitée au sein des populations pour le traitement du paludisme. Cassia sieberiana et Psydrax acutifolia suivent avec respectivement $60 \%$ et $54,7 \%$ de citations. Selon $47,5 \%$ de citations, les écorces de Parkia biglobosa sont utilisées dans le traitement des hémorroïdes.

\section{Vulnérabilité relative des espèces ligneuses}

$\mathrm{Au}$ total, 60 espèces possèdent un indice de vulnérabilité supérieur ou égal à 2 et sont de ce fait dites vulnérables. Parmi ces 60 espèces ,15 sont très vulnérables $(I V \geq 2,5)$. Diospyros mespiliformis, Sterculia setigera, Anogeissus leiocarpa, Detarium microcarpum, Khaya senegalensis et Prosopis africana sont les six prémières espèces les plus vulnérables. Treize espèces apparaissent faiblement vulnérables (Figure 5). Parmi les catégories (paramètres) de vulnérabilité, le mode de collecte est la principale cause de vulnérabilité des espèces ligneuses. En effet, $91,78 \%$ des espèces utilisées sont entièrement abattues, ou intensement écorcées. C'est le cas des prélèvements des écorces de Khaya senegalensis et de Sterculia setigera; de l'abattage des individus de Diospyros mespiliformis, Prosopis africana, Detarium microcarpum et Anogeissus leiocarpa. $72,60 \%$ des espèces utilisées sont faiblement représentées dans les formations végétales. $57,53 \%$ des espèces utilisées sont vulnérables à causes de la nature des organes prélévés. C'est le cas du bois de Detarium microcarpum, de Prosopis africana et de Pterocarpus erinaceus, des écorces de Khaya senegalensis et de Piliostigma thonningii, des calices de Bombax costatum, et des tiges de Saba senegalensis, etc. 16,44\% des espèces utilisées sont en partie très vulnérables en raison de leur préférence au sein des populations. Ces espèces sont Sterculia setigera, Diospyros mespiliformis, Anogeissus leiocarpa, Prosopis africana, Detarium microcarpum, Khaya senegalenis, Bombax costatum. Le nombre accru des domaines d'utilisations de certaines espèces les rend très vulnérables. C'est le cas de Piliostigma thonningii qui intervient dans cinq utilisations différentes (artisanat, construction, énergie, fourrage et medicinale).

\section{Perceptions locales et causes de dégradations des ressources végétales}

De façon générale, les interviewés constatent une diminution de la phytodiversité au cours des vingt dernières années. En effet, $52 \%$ des interviewés considèrent que les espèces ligneuses sont en régression. L'avis est plus optimiste chez d'autres $(29,6 \%)$ qui estiment que les ressources sont stables. Une partie non négligeable des interviewés $(18,4 \%)$ disent n'avoir aucune opinion. Toutefois, selon 53,5\% des citations, Pterocarpus erinaceus, Faidherbia albida, Afzelia africana, Hymenocardia acida, Khaya senegalensis, Securidaca longepedunculata, Sterculia setigera, Bombax costatum, Prosopis africana, Lophira lanceolata, Combretum molle, Pericopsis laxiflora, Diospyros mespiliformis, Detarium microcarpum, Ficus sycomorus, Vitex doniana, Sclerocarya birrea sont des espèces qui sont devenues rares. En se référant à l'indice de rareté, onze espèces parmi celles citées par les Sénoufo présentent un RI > $80 \%$, confirmant la rareté de plus de $64 \%$ des espèces perçues comme étant rares par les populations. Il s'agit de Lophira lanceolata (RI $=97,06 \%)$, Ficus sycomorus $(\mathrm{RI}=$ 94,12\%), Faidherbia albida, Khaya senegalensis, Sclerocarya birrea, Securidaca longepedunculata avec RI $=91,18 \%$, Diospyros mespiliformis Sterculia setigera avec RI $=85,29 \%$, Hymnocardia acida $(\mathrm{RI}=$ $88,24 \%)$, Bombax costatum et Vitex doniana avec RI $=82,35 \%$. Par ailleurs, en se basant sur l'importance écologique des espèces rares selon la perception locale, leurs Indices de Valeur d'Importance sont également faibles dans les formations végétales. C'est le cas de 
Lophira lanceolata (IVI = 0,29), Ficus sycomorus (IVI = 0,68), Khaya senegalensis $($ IVI $=2,53)$ (Tableau 4). Certaines espèces comme Afzelia africana, Detarium microcarpum, Pericopsis laxiflora, Prosopis africana sont moyennement présentes, alors qu'elles sont dites rares par les populations. Seule Pterocarpus erinaceus ( $\mathrm{RI}=55,88 \%$; $\mathrm{IVI}=13,19$ ) est fréquente dans les formations végétales.

Pour justifier la diminution des ressources, les informateurs Sénoufo évoquent plusieurs raisons que sont la croissance démographique, l'agriculture, la baisse de la pluviométrie. Des causes magico-religieuses sont également évoquées, en l'occurrence l'abandon des traditions ancestrales qui serait à l'origine de la baisse pluviométrique.

\section{Stratégies de conservation}

Pour ce qui est des méthodes de conservation, nos observations de terrain, nous ont permis de constater que les Sénoufo n'ont pas la culture de conserver les espèces locales dans leurs champs, en dehors de Vitellaria paradoxa, Parkia biglobosa et de Tamarindus indica qui leur sont directement utiles. Ce faisant, ces espèces sont maintenues et entretenues dans les champs. Cependant, les Sénoufo réalisent des plantations de certaines espèces exotiques. Il s'agit d'essences qui sont principalement à usage alimentaire (Anacardium occidentale, Mangifera indica) de bois de chauffe (Tectona grandis, Eucalyptus camaldulensis), de bois de construction (Eucalyptus camaldulensis).

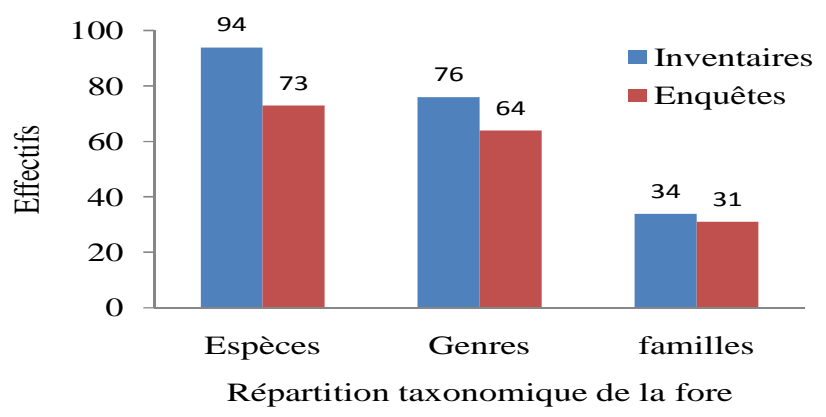

Figure 3: Spectre comparé des taxons sollicités dans les usages et ceux inventoriés dans les formations végétales.

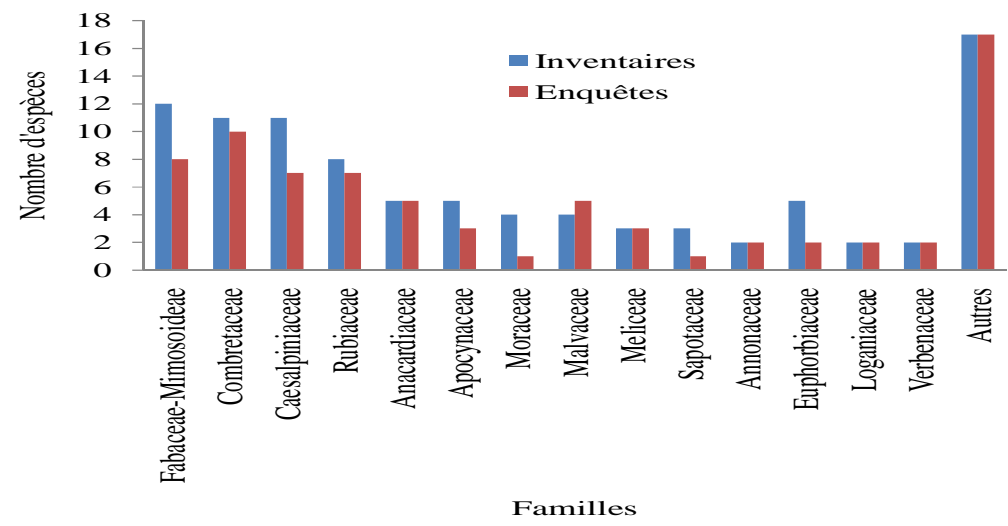

Figure 4: Spectre comparé des familles utilisées pour les besoins quotidiens et celles constitutives des formations végétales. 
Tableau 2: Les differentes utilisations des espèces inventoriées lors des enquêtes

\begin{tabular}{|c|c|c|c|c|c|c|c|}
\hline Espèces & Noms locaux & Alime & Artis & Const & Energ & Fourr & Médic \\
\hline Acacia dudgeoni & gonkoting (S) & & br & br & & & \\
\hline Adansonia digitata & Sienting (S) & pu,gra & & & & & \\
\hline Afzelia africana & Tchegui (S) & & & & & fe & \\
\hline Anacardium occidentale & - & $\mathrm{fr}$ & & & bo & & \\
\hline Annona senegalensis & namourog $(\mathrm{S})$ & $\mathrm{fr}$ & & & & fe & fe \\
\hline Anogeissus leiocarpa & Daguèlèg (S) & & & bo & bo & & fe \\
\hline Anthocleista procera & Dasunipaga (S) & & & & & & fe \\
\hline Blighia sapida & Kouguet (S) & $\mathrm{fr}$ & & & & & \\
\hline Bombax costatum & Sidalag (S) & $\mathrm{ca}$ & bo & & & & \\
\hline Bridelia ferruginea & Lokroting (S) & & & & & fe & \\
\hline Canarium schweinfurthii & Sian (D) & $\mathrm{fr}$ & & & & & \\
\hline Carapa procera & - & gr & & & & & \\
\hline Carica papaya & Manitchièg (S) & $\mathrm{fr}$ & & & & & \\
\hline Carissa edulis & Kabouting (S) & fr & & & & & \\
\hline Cassia sieberiana & Sidjanfi (D) & & & & & & fe,ra \\
\hline Ceiba pentandra & - & fe & & & & & \\
\hline Cola cordifolia & woding (S) & $\mathrm{fr}$ & & & & & \\
\hline Combretum collinum & Nouforwan (S) & & & & bo & & \\
\hline Combretum glutinosum & Djadjona (D) & & & & bo & & \\
\hline Combretum molle & Kardjam (S) & & & & & & fe \\
\hline
\end{tabular}


Combretum nigricans

Cordia myxa

Daniellia oliveri

Detarium microcarpum

Dichrostachys cinerea

Diospyros mespiliformis

Elaeis guineensis

Entada africana

Eucalyptus camaldulensis

Faidherbia albida

Ficus sycomorus

Gardenia aqualla

Gardenia erubescens

Gardenia ternifolia

Grewia bicolor

Guiera senegalensis

Hannoa undulata

Hymnocardia acida

Isoberlinia doka

Khaya senegalensis

Landolphia heudelotii

Lannea microcarpa

Lannea velutina

Lophira lanceolata doting (S)

Sourding (S)

Gbakoroting (S)

-

Yatcho (S)

Kanli (S)

Yirwag (S)

Tchatcharia (S)

Nichanting (S)

Kadjergue (S)

Katcharag (S)

Tèkèpou (S)

Yerting (S)

tchiordjenienne $(\mathrm{S})$

Tam (S)

Welding (S)

Davèrèting $(S)$

n'beku (D)

Fedouting (S)

Souanding (S) fr éc

bo

fe,fr

bo

bo

bo

bra

éc 
L. TRAORE et al. / Int. J. Biol. Chem. Sci. 5(1): 258-278, 2011

Maranthes polyandra

Mitragyna inermis

Parinari curatellifolia

Parkia biglobosa

Pavette crassipes

Pericopsis laxiflora

Piliostigma thonningii

Prosopis africana

Psydrax acutiflora

Pteleopsis suberosa

Pterocarpus erinaceus

Saba senegalensis

Sarcocephalus latifolus

Sclerocarya birrea

Securidaca longepedunculata

Sorindeia juglandifolia

Sterculia setigera

Strychnos spinosa

Tamarindus indica

Tectona grandis

Terminalia avicennioides

Terminalia laxiflora

Terminalia macroptera

Trichilia emetica

Uvaria chamae

Fanamourog (S)

Debroting (S)

Nèding $(S)$

Djim (S)

$\mathrm{pu}, \mathrm{gr}$

Tchianding (S)

Siannèrè $(\mathrm{S})$

Tiliougou (S)

Noufouyarg (S)

Dabiting (S)

Gonitchorog (S)

Zeloting (S)

Felep (S)

Grougrou (S)

Kassamgtoting (S

tomi (D)

$\operatorname{Dagog}(\mathrm{S})$

Nagoding (S)

Vernonia colorata

Kagbargui (S)

korsafinè (D)

Vitellaria paradoxa

loding/dogui (S)

Vitex doniana

Yakoro (S)

Kala (S)

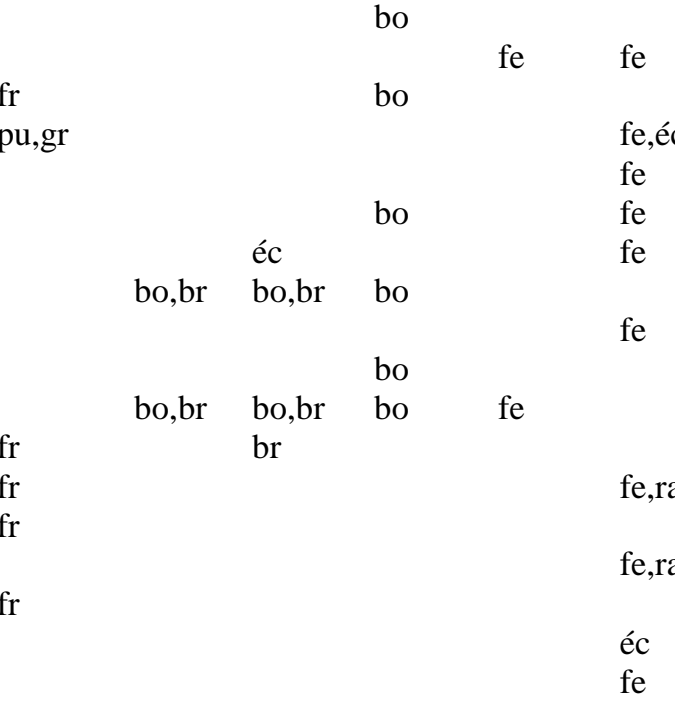

Zanthoxylum zanthoxyloides

Kala (S)

, $;$ Const $=$ Construction $;$ Energ $=$ Energie $;$ Fourr $=$ Fourrage $;$ Médic $=$ Médicinale $\mathrm{am}=$ amande $;$ boi $=$ bois $; \mathrm{br}=$ branches $;$ éc =écorces $; \mathrm{fe}=$ feuilles $; \mathrm{fr}=$ fruits $; \mathrm{gr}=$ graines $; \mathrm{pu}=$ pulpe. 
Tableau 3: Les espèces ligneuses interdites dans la combustion et leur pourcentage de citations chez les Sénoufo.

\begin{tabular}{lcccccc}
\hline Raisons d'interdiction & totem ou sacré & $\begin{array}{c}\text { Détruit la } \\
\text { famille }\end{array}$ & $\begin{array}{c}\text { raison } \\
\text { inconnue }\end{array}$ & $\begin{array}{c}\text { Ne brûle pas } \\
\text { bien }\end{array}$ & $\begin{array}{c}\text { Perte de la } \\
\text { fécondité }\end{array}$ & $\begin{array}{c}\text { Interdit par les ancêtres } \\
\text { lieu de culte }\end{array}$ \\
\hline Afzelia africana & 53,33 & - & - & - & - & 11,11 \\
Annona senegalensis & 6,67 & - & 11,11 & - & - & - \\
Bombax costatum & - & - & - & 31,11 & - & - \\
Ceiba pentandra & - & - & - & 22,22 & - & - \\
Combretum molle & - & 97,78 & - & - & - & - \\
Daniellia oliveri & - & - & 8,89 & - & - & - \\
Dichrostachys cinerea & 8,89 & - & 11,11 & - & - & - \\
Diospyros mespiliformis & - & - & 11,11 & - & - \\
Ficus sycomorus & - & - & - & 17,77 & - & - \\
Gardenia aqualla & - & - & 13,33 & - & - \\
Gardenia erubescens & - & - & 13,33 & - & - \\
Gardenia ternifolia & - & - & 13,33 & - & - \\
Guiera senegalensis & - & - & - & 20 & - \\
Zanthoxylum zanthoxyloides & - & - & 20 & - & - \\
\hline
\end{tabular}




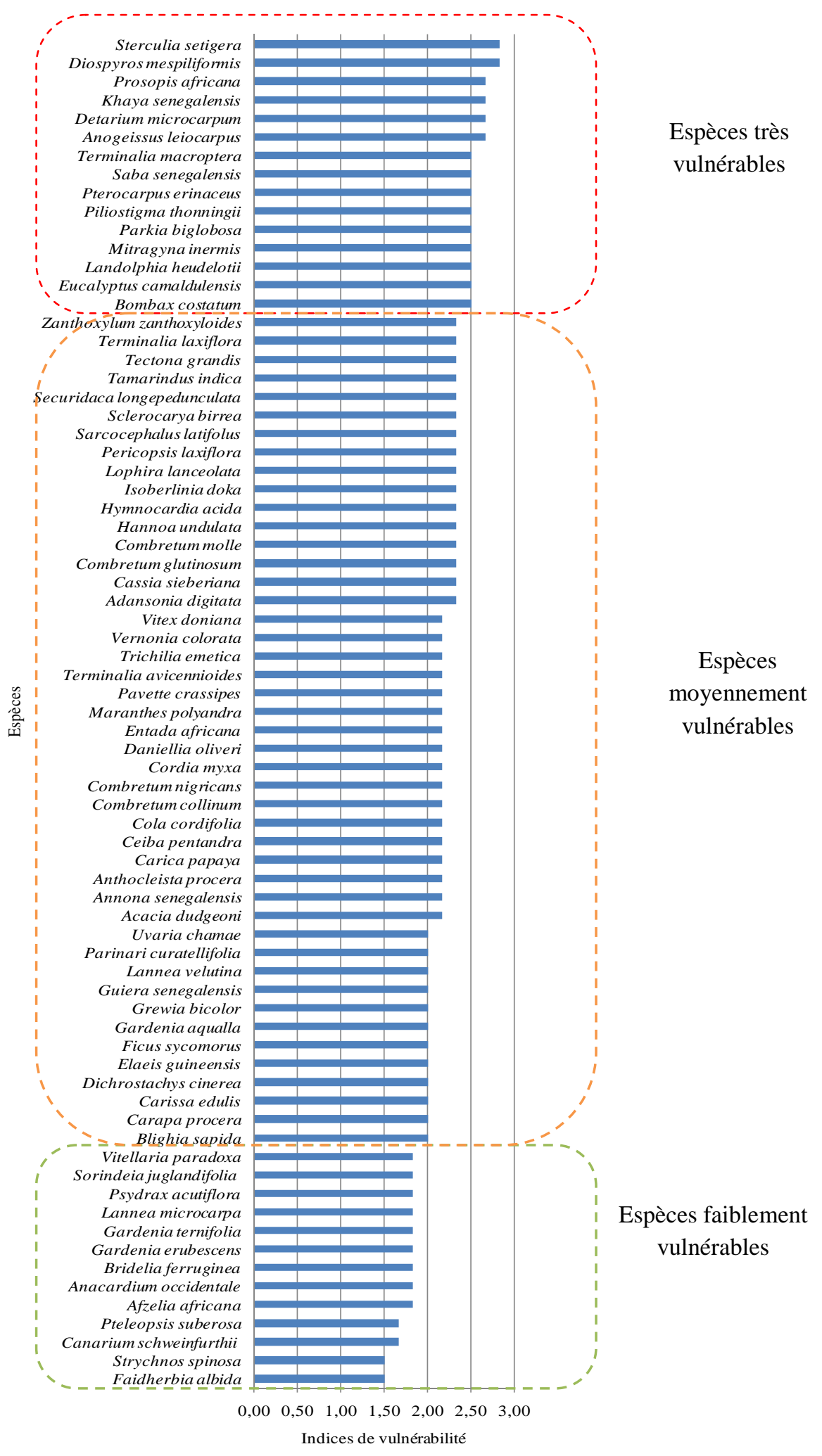

Figure 5: Spectre de vulnérabilité des espèces. 


\section{DISCUSSION}

Les utilisations

L'analyse floristique révèle une concordance entre les espèces citées et celles obtenues à partir des inventaires dendrométriques. Ce qui traduit d'une part la bonne connaissance que les populations ont de leur environnement, et d'autre part la fiabilité des enquêtes ethnobotaniques comme moyen pour une estimation rapide de l'état de la végétation d'une localité. Le plus grand pourcentage $(30,58 \%)$ d'utilisation du bois montre non seulement sa constante disponibilité en toute période de l'année, mais aussi sa forte demande dans plusieurs commodités d'usage que sont l'artisanat utilitaire, l'énergie, la construction des maisons, des hangars pour le séchage des céréales. Le pourcentage d'utilisation assez élevé des feuilles $(23,14 \%)$ pourrait s'expliquer par leur accessibilité plus facile mais aussi par leur importance pour divers usages tels que l'alimentation, le fourrage et la pharmacopée. A titre d'exemple, les feuilles ont une grande proportion de citations parmi les organes utilisés dans les traitements de toutes les maladies énumérées. Le choix des espèces résistantes est déterminant pour la durée des constructions. Ainsi, les critères de choix des espèces sont la dureté du bois, la résistance aux insectes foreurs. Ces critères de choix sont rapportés par Thiombiano et al. (2002), Lykke et al. (2004), Krohmer et al. (2006) et Bognounou (2009). Se référant toujours aux travaux de ces auteurs, Anogeissus leiocarpa est préférée en construction pour la consistance dure et la résistance de son bois aux termites.

Dans le domaine de l'énergie, le bois demeure le principal combustible des ménages dans le milieu rural. Toutes les sources sont unanimes pour confirmer que l'usage du boisénergie revêt une importance majeure pour les pays en voie de développement et pour les pauvres en dehors de ces pays (Pearce, 2001). Cette forte dépendance vis-à-vis des ressources végétales pour les besoins énergétiques se traduit chez les Sénoufo par une utilisation de la plupart des ligneux des formations végétales à l'exception de celles interdites. L'appréciation de la qualité énergétique des espèces est relative et varie en fonction des localités et des groupes ethniques. Ainsi au Sahel, Guiera senegalensis occupe le premier rang parmi les espèces préférées pour le bois de chauffe dans la province du Yagha (Ganaba et al., 1998), tandis qu'elle est rejetée chez les Sénoufo à cause de ses mauvaises qualités énergétiques. Bien que des disparités existent dans l'appréciation de la qualité énergétique de certains ligneux, certaines espèces par contre semblent faire l'unanimité en termes de préférences au sein des populations du Burkina Faso et de la sous région Ouest africaine. En effet, les qualités énergétiques de Anogeissus leiocarpa, Combretum glutinosum, Prosopis africana et de Pterocarpus erinaceus sont mentionnées par de nombreux auteurs (Brondeau, 2001; Thiombiano et al., 2002, Weber et al., 2008; Folefack et Abou, 2009 ; Bognounou, 2009).

Plusieurs espèces ont été citées par les Sénoufo comme interdites en combustion. Combretum molle est le «totem » commun. Son nom local « kardjam » signifie « détruire la famille ». Le bois de l'espèce ne doit pas être brûlé dans la famille. Elle peut être brûlée en dehors du village. C'est une des constantes pour les espèces sacrées qui est que leur bois ne doit pas être brûlé au village, mais uniquement en brousse, lorsque cela est vraiment nécessaire (Ouédraogo-Belem, 2008). D'autres raisons seraient liées au fait que les ancêtres des Sénoufo n'utilisaient pas ces espèces pour le bois de chauffe. Des raisons similaires sont rapportées par Kristensen and Balslev (2003) chez les Gourounsi au Sud du Burkina Faso où certaines espèces sont également rejetées en combustion. Selon Taïta (2003), Afzelia africana et Diospyros mespiliformis sont interdites en combustion chez les Bobo. Les critères prédominants de rejet des espèces ligneuses comme bois de feu sont les croyances religieuses ancestrales (Ganaba et 
al., 1998). L'interdiction des espèces ligneuses dans la combustion semble être répandue en Afrique. Les travaux de Smith et al., (1996) et Lykke (2000) ont mentionné l'existence de ligneux interdits en combustion, en Tanzanie et au Sénégal. Les coutumes et croyances qui rejettent l'utilisation de certaines espèces contribuent à la conservation des ressources végétales.

La zone sahélo-soudanienne est caractérisée par l'existence d'une période où le fourrage herbacé devient rare. En ce moment, les ligneux offrent en permanence un fourrage vert. La cueillette se fait par émondage ou étêtage des arbres et arbustes. Afzelia africana est l'une des espèces fourragères préférées bien qu'elle soit sacrée chez les Sénoufo. Cela peut paraître paradoxal, mais montre que le caractère sacré est relatif à un usage. La perception de cette espèce est similaire chez les Bobo de la région des Haut Bassins du Burkina Faso. Selon Millogo-Rasolodimby (1993), l'espèce est crainte car elle est considérée comme l'habitat favori des génies. Ainsi, l'arbre n'est jamais abattu à la hache ; la base du tronc est induite de poudre inflammatoire suivant un anneau afin que le feu puisse ronger petit à petit cette partie induite jusqu'à sa mort. L'utilisation de l'arbre mort soit pour le bois de chauffe, soit pour les meubles, soit pour la fabrication des caisses de résonnance des tambours, est permise en ce moment. En principe, si une espèce est dite sacrée, elle ne devrait être utilisée sous aucune autre forme ou sous aucun prétexte. Mais cela n'est pas le cas de Afzelia africana. Dans ces conditions, la notion de sacrée est relative et semble dépendre de l'usage dans lequel l'espèce est interdite, de l'état dans lequel le prélèvement est fait, de la disponibilité même des ressources végétales pour les usages que l'on veut en faire.

Ce qui peut justifier le choix des espèces en artisanat est certainement la valeur et l'importance sociales de l'objet fabriqué. Le mortier et le pilon n'ont pas la même valeur que le tabouret. En effet, le mortier étant une vase dans laquelle les céréales sont pilées, doit être en principe confectionné à partir d'un bois dur et résistant, alors qu'un simple morceau de tronc d'arbre peut valablement servir à confectionner le tabouret.

Dans le domaine de l'alimentation, de nombreuses espèces produisent des fruits comestibles. Le nombre assez important de fruits cités montre leur importance dans l'alimentation des populations. Des espèces comme Vitellaria pardoxa et Parkia biglobosa sont très appréciées. La disponibilité de la plupart des fruits coïncide avec le début des travaux champêtres et de la baisse des provisions de denrées alimentaires (Kristensen and Balslev, 2003). En ce moment, la disponibilité et la consommation des fruits sauvages constituent un apport considérable dans l'alimentation des ménages puisque selon Guinko et Pasgo (1992), les fruits sauvages contribuent à une alimentation variée en termes d'apport de vitamines. La consommation des fruits de la plupart des espèces citées par les Sénoufo est confirmée par les travaux de certains auteurs (Ambé, 2001 ; Kristensen and Balslev, 2003 ; Taïta, 2003).

Dans le domaine de la médecine traditionnelle, les remèdes du paludisme et des maux de ventre ont été les plus cités par les populations. Les citations fréquentes des remèdes de ces maladies sont dues à leur haute prévalence au sein des populations. Selon l'O.M.S (2002), le paludisme est la principale cause de mortalité chez les enfants de moins de cinq ans en Afrique (20\%) et il représente $10 \%$ de la charge totale de morbidité du continent. Cette réalité étant vécue notamment par les populations rurales, semble être la raison probable qui fait que les remèdes du paludisme ne sont pas entourés de grands secrets. Pour ces populations, toutes les plantes soignent des maladies. Selon elles, les prélèvements à des fins thérapeutiques dépendent des incantations que l'on prononce. Faire des incantations constitue l'une des multiples facettes de la pratique de la médecine traditionnelle (Benoît, 2008). Ces 
incantations sont une forme de jeu de mots prononcés oralement pour accroître l'efficacité du médicament (Capes/rgc-b, 2006). Cette perception de la médecine traditionnelle, s'inscrit dans un constat général rapporté par Ouôba et al. (2006), à savoir que la médecine traditionnelle est un domaine relativement fermé où le savoir sur les plantes médicinales se transmet de père en fils.

\section{Disponibilité des ressources forestières et les causes de leur dégradation}

La perception des Sénoufo sur l'état des ressources concorde avec les constats faits sur le terrain. En effet, plus de 50\% des espèces citées par les Sénoufo comme étant des espèces rares, font partie des espèces rares révélées par le calcul de l'indice de rareté à l'issue des inventaires. Celles qui sont moyennement fréquentes dans les formations végétales, présentent également des indices de valeurs d'importance assez faibles. Il est fort probable que cette faible importance écologique et l'importance socio-économique des espèces aient pesé dans l'appréciation par les Sénoufo qui les citent parmi les espèces rares. Au regard de leur importance socio économique, une moindre variation au niveau de la disponibilité de certaines espèces ne peut passer inaperçu. C'est pourquoi la perception locale est aussi indispensable pour une meilleure appréciation de l'état de la végétation, surtout pour l'identification des espèces menacées de disparition (Lykke, 2000). Mais de nombreuses espèces, parmi lesquelles Andira inermis, Antiaris africana, etc. (Tableau 4), n'ont pas été mentionnées par les Sénoufo comme espèces rares, alors que dans la réalité, elles sont très rares. Cela évoque l'intérêt direct que les populations locales accordent à une espèce végétale. C'est d'ailleurs pourquoi dans une étude semblable, Hahn-Hadjali et Thiombiano (2000) ont constaté que les populations évoquent ou remarquent seulement les espèces qui leur sont utiles, celles qui ne sont pas utilisées passent inaperçues et peuvent même disparaître sans qu'ils ne s'en aperçoivent.
Dans l'ensemble, si la population est consciente de la dégradation des ressources végétales, les causes de cette dégradation ne sont pas toujours bien perçues. C'est pourquoi, en plus des causes réelles (pression anthropique, aléas climatiques), les causes magico-religieuses ont été évoquées par les Sénoufo. Les causes magico-religieuses comme facteurs explicatifs des changements environnementaux ne sont pas spécifiques aux Sénoufo. En effet, le déficit pluviométrique, selon la population Mossé de la Région du Centre et du Plateau central au Burkina Faso, est dû au non respect des coutumes et traditions (Benoît, 2008).

Vulnérabilité et impact des usages sur la disponibilité des ressources forestières

L'action anthropique semble jouer un rôle majeur dans la destruction du couvert végétal dans le Sud-Ouest. Les paysans abattent chaque année des superficies importantes de brousse pour la culture du coton qu'ils abandonnent l'année suivante à la recherche d'autres terres. Lors des défriches, les espèces utilitaires, tant alimentaires, artisanales, fourragères, énergétiques que médicinales ne sont pas épargnées, contribuant ainsi à la dégradation de la biodiversité. Les quelques reliques boisées, qui ont été épargnées lors de l'installation de champs et les écosystèmes (inselbergs) impropres aux activités agricoles, subissent désormais la pression du prélèvement des produits végétaux. La pression anthropique accentue la réduction des habitats, du fait de la faiblesse des ressources et l'effectif de la population sans cesse croissante. Pour ce faire le prélèvement du bois, des écorces, des racines, des feuilles, des fleurs ou autres organes végétaux prennent de l'ampleur et accentuent ainsi la vulnérabilité des espèces. La récurrence des prélèvements affaiblit les individus ligneux et les rend vulnérables aux intemperies (vent, feux, sécheresse) et aux attaques parasitaires. Le mauvais état sanitaire qui en résulte, se traduit par un dessèchement partiel ou total des individus. Ces effets 
négatifs qui causent la baisse de densité et la perte de la diversité floristique des populations ligneuses sont vite perceptibles au niveau des espèces ligneuses qui constituent la composante permanente des savanes du pays. De façon générale, le degré de pertubation de la population d'une espèce et la vulnérabilité de cette dernière dépendent de la demande, de l'offre (le disponible ligneux), des parties utilisées et du type de croissance (Cunningham, 1996). Par ailleurs, en analysant l'impact des prélèvements de produits ou organes végétaux, Taïta (2003) fait remarquer que les espèces dont les fruits et les graines sont consommés ou utilisés à d'autres fins peuvent avoir des problèmes de dissemination et pire, la récolte de certains fruits à l'état vert influe négativement sur la pérennité de l'espèce concernée (OuédraogoBelem, 2008). C'est le cas de Diospyros mespiliformis qui est une espèce rare et dont les fruits verts sont utilisés dans la poterie. Il existe donc une relation manifeste entre la partie exploitée d'une plante et les effets de cette exploitation sur la plante elle- même (Cunningham, 1996). La vulnérabilité de certaines espèces dans le Sud-Ouest s'explique aussi par le fait qu'elles sont très sollicitées dans plusieurs domaines d'utilisation particulièrement en énergie, en construction et en artisanat utilitaire, dont le principal mode d'exploitation demeure une destruction partielle ou totale des individus. C'est le cas de Prosopis africana, et Pterocarpus erinaceus. La sollicitation des espèces dans plusieurs domaines d'utilisation à la fois peut constituer une raison de forte vulnérabilité. Par ailleurs, les usages en médecine traditionnelle qui concernent le prélèvement des écorces et des racines causent d'énormes dommages aux espèces végétales. En effet, selon Hahn-Hadjali et Thiombiano, (2000) l'écorçage intensif entraîne une perte de vigueur des arbres. C'est dire que les espèces victimes de ce mode de prélèvement, n'arrivent plus à assurer leurs fonctions physiologiques au mieux de leurs potentialités. Cette situation de troubles physiologiques influence nécessairement la production des fruits et partant celle des graines qui assurent la pérennité de l'espèce.

\section{Conclusion}

La présente étude a permis d'appréhender les valeurs accordées aux ressources végétales ligneuses par les Sénoufo, notamment leur importance socioéconomique. Les usages et les perceptions ont permis de montrer toute l'importance des croyances traditionnelles et ancestrales dans la gestion des ressources naturelles. La variation des choix et des préférences observées dans l'utilisation des espèces ligneuses est liée aux croyances ancestrales et à la disponibilité des ressources forestières.

$\mathrm{Si}$ quelques stratégies de conservation sont entreprises par le maintien et l'entretien de certaines espèces de grande importance, très peu d'actions significatives sont entreprises pour lutter efficacement contre la dégradation des ressources végétales. Bien que les populations soient conscientes de l'importance des espèces locales dans leur vie quotidienne, elles n'ont pas la culture de les conserver grâce à des plantations. Il faudra alors un changement de comportement. Cela passe par un travail de sensibilisation sur la nécessité de produire ces espèces locales, tout au moins celles que les populations jugent prioritaires et qui sont adaptées à leur milieu. C'est dans ce sens que les espèces préférées doivent faire l'objet de promotion de la part des agents de développement. Les espèces qui sont moins importantes, parce que peu utilisées, ont moins de chance d'épouser l'assentiment des populations. Malgré leur moindre importance, ces espèces dites de moindre valeur doivent être également protégées pour les besoins de la conservation de la diversité biologique. Cela est d'autant plus vrai qu'une espèce moins importante de nos jours pourrait être recherchée plus tard en remplacement d'autres, qui deviennent rares, dans un domaine d'utilisation.

L'évaluation de la vulnérabilité des ressources forestières a permis de dresser une 
liste d'espèces ligneuses vulnérables dans le Sud-Ouest du Burkina Faso; il convient toutefois de noter qu'elle est relative et non exhaustive. En effet, il est important de reconnaître que les populations évoquent les usages courants ou remarquent seulement les espèces qui leur sont utiles ; celles qui ne sont pas couramment utilisées peuvent même être très vulnérables sans que l'on ne s'en aperçoive. A ce titre, les données d'inventaires restent très utiles pour avoir une appréciation fiable et complète de la disponibilité des ressources.

\section{REMERCIEMENTS}

Nous remercions vivement le ministère fédéral allemand de l'Éducation et de la Recherche (BMBF) qui a financé cette étude, à travers le Programme BIOTA W11, ainsi que les populations Sénoufo du Sud-Ouest pour leur disponibilité lors de travaux de terrain, et les experts qui ont évalué cet article.

\section{REFERENCES}

Adomou AC. 2005. Vegetation patterns and environmental gardients in Bénin. Implications for biogeography and conservation. $\mathrm{PhD}$ thesis Wageningen University, Wageningen, p.132.

Ambé GA. 2001. Les fruits sauvages comestibles des savanes guinéennes de Côte-d'Ivoire: état de la connaissance par une population locale, les Malinké. Biotechnol. Agron. Soc. Environ., 5(1): 43-58.

Belem B, Nacoulma BMI, Gbangou R, Kambou S, Hansen HH, Gausset Q, Lund S, Raebild, A, Lompo D, Ouédraogo M, Theilade I, Boussim IJ. 2007. Use of None Wood Forest Products by local people bordering the "Parc National Kaboré Tambi", Burkina Faso. The Journal Transdisciplinary Environmental Studies, 6(1): 1-21.

Belem B, Olsen CS, Theilade I, Bellefontaine R, Guniko S, Lykke AM, Diallo A, Boussim JI. 2008. Identification des arbres hors forêt préférés des populations du Sanmatenga (Burkina Faso). Bois et Forêts des Tropiques, 298(4): 53-64.

Benoît E. 2008. Les changements climatiques: vulnérabilité, impacts et adaptation dans le monde de la médecine traditionnelle au Burkina Faso. VertigO, 8(1): 1-12.

Betti JL. 2001. Vulnérabilité des plantes utilisées comme antipaludiques dans l'arrondissement de Mintom au sud de la réserve de Biosphère du $\mathrm{Dja}$ (Cameroun). Syst. Geogr. Pl., 71: 661-678.

Bognounou F, Thiombiano A, Savadogo P, Boussim JI, Odén PC, Guinko S. 2009. Woody vegetation structure and composition at four sites along latitudinal gradient in Western Burkina Faso. Bois et Forêt des Tropiques, 300(2): 29-44.

Brondeau F. 2001. Evolution de la filière bois énergie et dynamique des formations ligneuses autour de l'Office du Niger. Bois et Forêt des Tropiques, 270(4): 1534.

CAPES/RGC-B (Centre d'Analyse des Politiques Economiques et Sociales/Réseau de Gestion des Connaissances au Burkina). 2006. Etat des Lieux des Savoirs Locaux au Burkina Faso : Ethnobotanique et Médecine Traditionnelle, Pratiques et Systèmes Culturaux, Ethnozoologie et Santé Animale, Habitats, Matériaux Locaux et Energie, Artisanat, Arts du Feu et Pratiques Funéraires. CAPES/RGC-B ; 379.

Cunningham AB. 1996. Peuples, Parc et Plantes. Recommandation pour les zones à usages multiples et les alternatives de développement autour du parc national de Bwindi Impénétrable. Ouganda. Document de travail Peuples et Plantes $\mathrm{n}^{\circ}$ 4. UNESCO ; Paris, p.64.

Folelack DP, Abou S. 2009. Commercialisation du bois de chauffe en zone sahélienne du Cameroun. Sécheresse, 20(3): 312-318.

Fontes J, Guinko S. 1995. Carte de la végétation et de l'occupation du sol du 
Burkina Faso. Notice explicative. Ministère de la Coopération française, projet Campus, Toulouse, p. 68.

Ganaba S, Ouadba JM, Bognounou O. 1998. Les ligneux à usage de bois d'énergie en région sahélienne du Burkina Faso: préférences des groupes ethniques. Sécheresse, 9: 261-268.

Guinko S, Pasgo L. 1992. Récolte et commercialisation des produits non ligneux des essences forestières locales dans le département de Zitenga, au Burkina Faso. Unasylva, 43(168): 16-19.

Guinko S. 1993. Les plantes et la médecine traditionnelle au Burkina Faso. Berichte des Sonderforschungsbereichs, 268, Bd. 1, Frankfurt a.M. : 47-53.

Hahn-Hadjali K, Thiombiano A. 2000. Perception des espèces en voie de disparition en milieu Gourmantché (Est du Burkina Faso). Berichte des Sonderforschungsbereichs, 268, Band 14, Frankfurt a.M. : 285-297.

INSD (Institut National de la Statistique et de la Démographie). 2008. Recensement Général de la Population et de l'Habitation de 2006, Résultats définitifs. INSD : Ouagadougou, Burkina Faso.

Kokou K, Adjossou K, Hamberger K. 2005. Les forêts sacrées de l'aire Ouatchi au Sud-Est du Togo et les contraintes actuelles des modes de gestion locales des ressources forestières. VertigO, 6(3): 110.

Kristensen M, Balslev H. 2003. Perceptions, use and availability of woody plants among the Gourounsi in Burkina Faso. Biodiversity and Conservation, 12: 17151739.

Krohmer J, Hahn-Hadjali K, Wittig R. 2006. Utilisation de la flore sauvage par des populations peules au Burkina Faso et Bénin. Etude sur la Flore et la Végétation du Burkina Faso et des Pays Avoisinants, 10: $33-48$

Millogo-Rasolodimby J. 1993. Les plantes sacrées chez les Bobos. Berichte des
Sonderforschungsbereichs, 268, Band 5, Frankfurt/Main : 93-100

OMS. 2002. The World Health Report, Geneva. http://www.who.int/whr/2002/.

Ouattara IZ. 2008. La conception traditionnelle de la mort à travers les chansons funéraires chez les Sénoufo de Kawolo. Mémoire de Maitrise, Université de Ouagadougou, p. 132.

Ouédraogo-Belem M. 2008. Les galeries forestières de la Réserve de la Biosphère de la Mare aux Hippopotames du Burkina Faso: caractéristiques, dynamique et ethnobotanique. Thèse de Doctorat d'Etat ès Sciences Naturelles, Université de Ouagadougou, p. 262.

Ouédraogo A. 2006. Diversité et dynamique de la végétation ligneuse de la partie orientale du Burkina Faso. Thèse de Doctorat unique, Université de Ouagadougou, p. 195.

Ouôba P, Lykke AM, Boussim J, Guinko S. 2006. La flore médicinale de la Forêt Classée de Niangoloko (Burkina Faso). Etude sur la Flore et la Végétation du Burkina Faso et des Pays Avoisinants, 10: 5-16.

Pearce DW. 2001. The Economic Value of Forest Ecosystems. Ecosystems Health, 7(4): 284-296.

Peters CM. 1997. Exploitation Soutenue de Produits Forestiers autres que le Bois en Forêt Tropicale Humide: Manuel d'Initiation Ecologique. Série générale du Programme d'Appui à la Biodiversité 2: 49p. WWW-NC-WRI/USAID.

Lykke AM. 2000. Local perception of vegetation change and priorities for conservation of woody-savanna vegetation in Senegal. Journal of Environmental Management, 59: 107-120.

Lykke AM, Kristensen MK, and Ganaba S. 2004. Valuation of local use and dynamics of 56 woody species in the Sahel. Biodiversity and Conservation, 13: 1961-1990.

Smith W, Meredith TC, Johns T. 1996. Use and conservation of woody vegetation by 
the Batemi of Ngorongoro district, Tanzania. Economic Botany, 50: 290-299.

Taïta P. 2003: Use of woody plants by locals in Mare aux Hippopotames Biosphere Reserve in western Burkina Faso. Biodiversity and Conservation, 12: 12051217.

Thiombiano A, Ouôba P, Guinko S. 2002. Place des Combretaceae dans la société gourmantché à l'est du Burkina Faso. Etude sur la Flore et la Végétation du Burkina Faso et des Pays Avoisinants, 7: 17-22.

Thiombiano A. 2005. Les Combretaceae du Burkina Faso: taxonomie, écologie, dynamique et regeneration des espèces.
Thèse de Doctorat d'Etat. Université de Ouagadougou, p. 290.

Weber JC, Larwanou M, Abasse TA, Kalinganire A. 2008. Growth and survival of Prosopis africana provenances tested in Niger and related to rainfall gradients in the West African Sahel. Forest Ecology and Management, 256: 585-592.

Wezel A , Haigis J. 2000. Farmer's perception of vegetation changes in semi-arid Niger. Land Degradation and Development, 11:523-534.

Wezel A, Lykke AM. 2006. Woody vegetation in Sahelian West Africa: evidence from local knowledge. Environ Dev Sustain, 8: 553-567. 